\title{
EXPERIENCE OF THE EROSION-CORROSION PROBLEMS IN THE MAIN RING COOLING WATER SYSTEM AT THE KEK-PS
}

\author{
Hikaru Sato, Tomio Kubo, Katsumi Marutsuka, Yoshio Saito, Masashi Shirakata and Noboru Tokuda \\ Accelerator Laboratory, High Energy Accelerator Research Organization (KEK), \\ Oho 1-1, Tsukuba-shi, Ibaraki-ken 305-0801, Japan
}

\section{Abstract}

After more than two decades have passed since the KEK-PS construction, water leak accidents in the cooling system have occurred frequently. Low conductivity deionized water, 20 degrees centigrade, is supplied at the Mechanical support building and circulates in a closed loop. Water flows through the main ring magnets, various magnets for beam injection and extraction, correction magnets and power supply system, and so on. Recently, various repair works due to the plugged water pipes have been tried with the endless job. We must do it in a high radio activated area. After several investigations, most of these come from the erosion-corrosion after the long time use. We will introduce the experience of the erosioncorrosion problems and lessons from these experience so as to design the cooling system and to select the materials.

\section{INTRODUCTION}

The KEK-PS complex comprises four accelerators: two 750-keV Cockcroft Walton pre-injectors, a 40-MeV injector linac, a $500-\mathrm{MeV}$ booster synchrotron and a 12$\mathrm{GeV}$ main ring. Slow extracted beams are served to two counter halls (north and east) by a half integer resonance, and the internal target has also served the secondary beam in the east counter hall. The long-base-line neutrinooscillation experiment, K2K, has started in January 1999 in the high intensity operation by the newly fast extraction.

However, we have experienced several type troubles on the cooling water system, such as flow switch blockage, pinhole leaks in the copper pipe, deterioration of the rubber tube etc., in the PS main ring magnet and power supply cooling system. These problems cause equipment failure and hinder the stable machine operation. Evidences of an erosion and/or corrosion problem were reported in several laboratories $[1,2,3]$.

There are five de-ionized cooling water systems (named P11, P20, P23, P24, and P25) and the pumping stations are housed in the mechanical support building.

In this report, some experience at the P11 and P20 cooling systems are described. The P11 loop supplies deionized water to the pre-injector, linac tanks, 40- and 500$\mathrm{MeV}$ beam transport line, booster magnets, main ring magnets and accelerating RF systems. De-ionized water in this system flows through the radio activated area. The P20 loop supplies also de-ionized water to the power supplies of booster and main ring.
A schematic of the P11 cooling system is shown in Fig. 1. The system comprises an expansion tank with nitrogen gas cylinder, two pumps which supply de-ionized water, two water purifiers (ion exchanger), a conductivity detector, a plate type heat exchanger with a chilled water pump, a three way mixing valve to control the water temperature and a tank type heat exchanger with a chilled water loop. Original de-ionized water is supplied to the expansion tank. Temperature of water through heat loads, such as linac, booster, main ring etc, is 39 degrees centigrade. The plate type heat exchanger cools the heated water to 27 degrees centigrade, and the tank type heat exchanger cools to 20 degrees centigrade. The flow rate is $4520 \mathrm{l} / \mathrm{min}$ and the pressure is $10.2 \mathrm{kgf} / \mathrm{cm}^{2}$. Five percent of circulating water is bypassed to the water purifier (ion exchanger) and the conductivity is monitored.

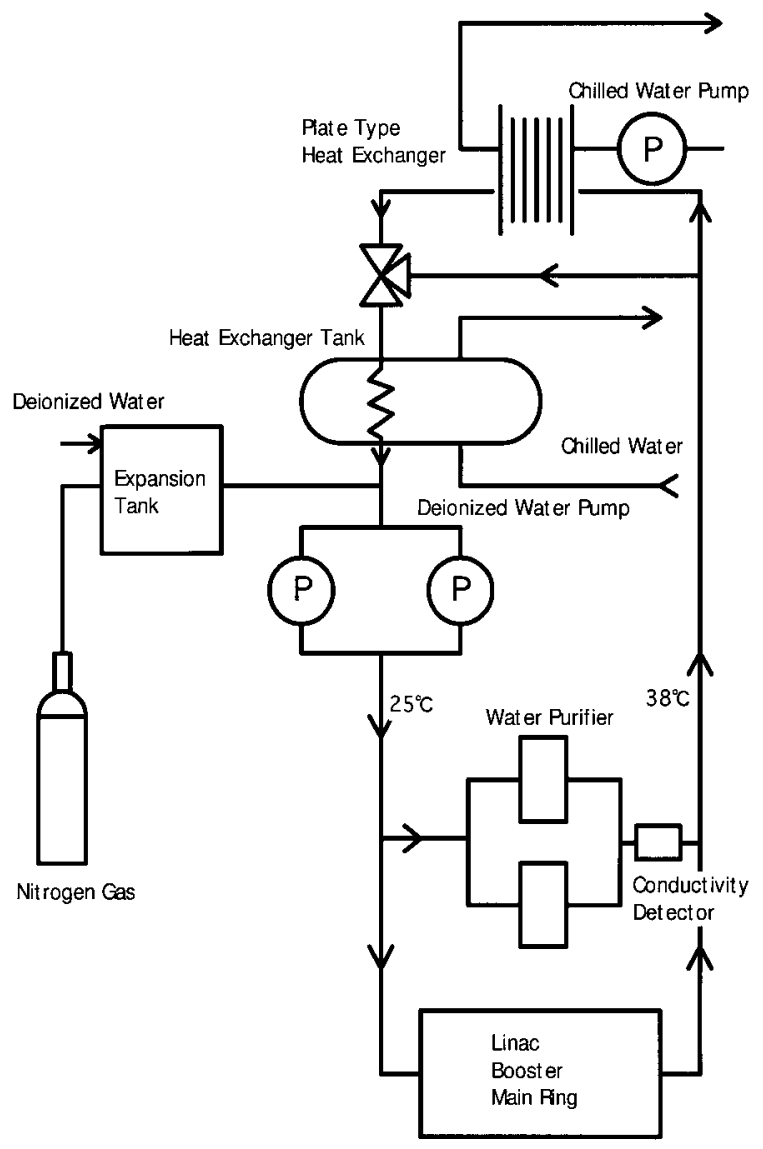

Figure 1: Schematic of the P11 cooling system. 
Conductivity is controlled at $0.1 \mu \mathrm{S} / \mathrm{cm}$ at the output of purifiers. In the P20 cooling system, a conductivity detector is equipped at the main ring power supply system to protect the high voltage thyristor converter. During stable runs, conductivity is maintained at $0.4 \mu \mathrm{S} / \mathrm{cm}$ and a protective relay acts at $5 \mu \mathrm{S} / \mathrm{cm}$. The materials of piping is made of SUS304TP in the mechanical support building, but other materials, such as brass, copper, iron, etc. are used in the accelerator. An operator of the mechanical support says there are no sludge in strainers to be washed out. In the main ring magnets and power supplies, however, we have ever washed strainers and valves many times.

\section{EXPERIENCE}

Blockage of the flow switch in the main ring magnetcooling pipe has occurred several times for the last two decades. When we had no time, we repaired temporarily by hitting flow switch or opening and closing valves repeatedly. During long machine shut down, we washed the troubled valves and strainers after cooling down of radioactivity.

In the sextupole magnet cooling system, the blockage trouble of the flow switch occurred frequently. Strainers had been never maintained until we washed all the strainers in November 1997. After that, no such trouble has occurred.

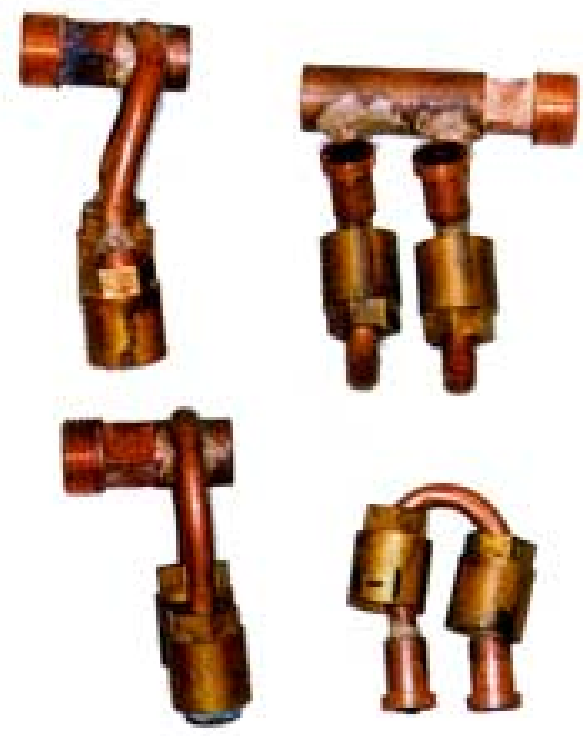

Figure 2: Old small piping parts for the quadrupole magnet cooling loop.

The flow switch of the specific magnet in the main ring bending magnet array has closed frequently and the troubles occurred after washing the strainer. We washed the strainer repeatedly and installed the flow meter to monitor the flow rate.

We inspected the sludge in the strainer by the chemical analysis. Main components are ethylene-propylene rubber and butylene one by the infrared rays absorption spectrum analysis. Fluorescent X-rays analysis detects iron, copper, chromium, nickel, zinc and a small amount of aluminum.

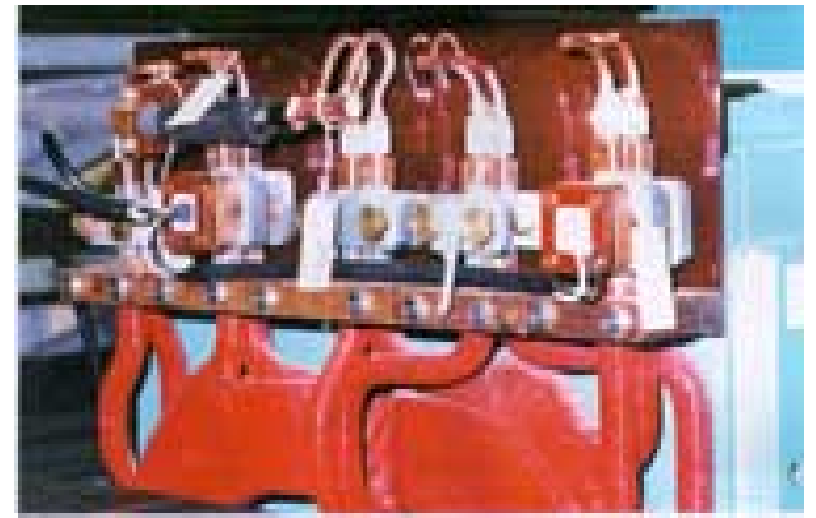

Figure 3: Replaced small piping parts at the upper half of the quadrupole magnet.

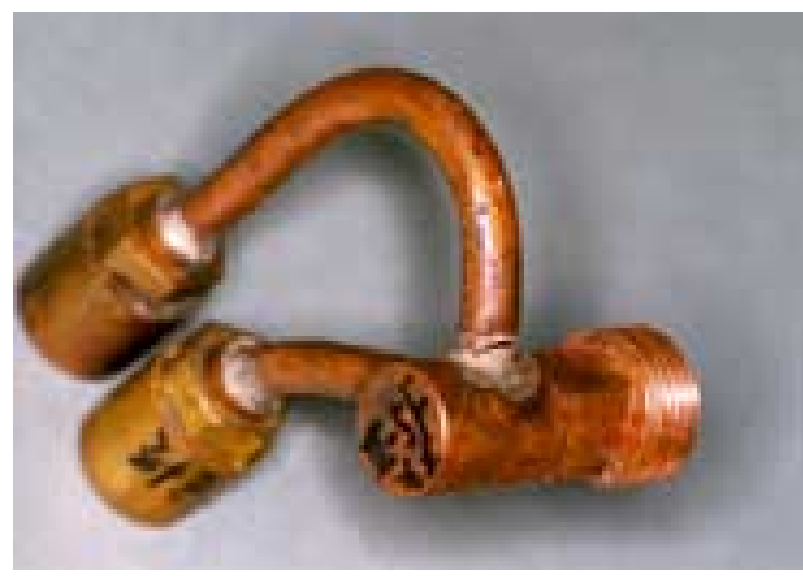

Figure 4: Broken small piping part.

A main ring quadrupole magnet has four water cooling loops, and they are concentrated to one pipe to connect the main cooling loop. Then, four types and ten small piping parts are equipped at the end of the quadrupole magnet. Figure 2 shows three type old parts (two of lefts are same type). They were replaced new ones as shown in Fig.3. These parts are made of copper pipe of $6 \mathrm{~mm}$ of outer diameter and $0.6 \mathrm{~mm}$ of thickness and brass. Pinhole leaks occurred on these pipes for recent years. Microscopic inspection the small piping parts indicates clearly the trace of erosion. The pipe wall became very thin and is $0.05 \mathrm{~mm}$ at the thinnest position. These phenomena indicate a possibility of erosion. Figure 4 shows a typical broken part. It is impossible to inspect the inner surface of these parts by cutting, since it is highly radioactivated. Therefore we inspected the same type parts of a field monitor magnet in the power supply building. We found an erosion trace (black colored copper oxide coat is stripped off) in most of the parts as shown typically in Fig. 5, 6. The flow speed of water is estimated as $6 \mathrm{~m} / \mathrm{s}$ in these pipes, and erosion is a matter of course.

Three quarters of these parts (especially in high radioactive area) were replaced during the summer shut down in 2000, as shown in Fig. 3. The remained parts will be replaced during the summer shut down in this year. 
The inside of hollow conductors of a quadrupole magnet for field monitor in P20 loop was inspected by the endoscopic examination. There were no heavy erosive surfaces, but several metallic luster points were found.

Rubber tubes for the main ring power supply cooling system have been replaced twice in the past because of the insulation resistance drop. During the summer shut down in 2000, the rubber tubes for the field monitorbending magnet were replaced. Since these parts were not activated, we could inspect them by chemical and spectrum analysis. Figure 7 shows an inner surface of this rubber tube. Adherent brown material was identified to waterweed, iron, copper, chromium, silver, zinc, nickel, lead and their oxide. The white luster material was identified to iron, copper, zinc, nickel and cobalt. We estimate that these are from various materials in the piping parts by erosion-corrosion and adhered on the surface of rubber.

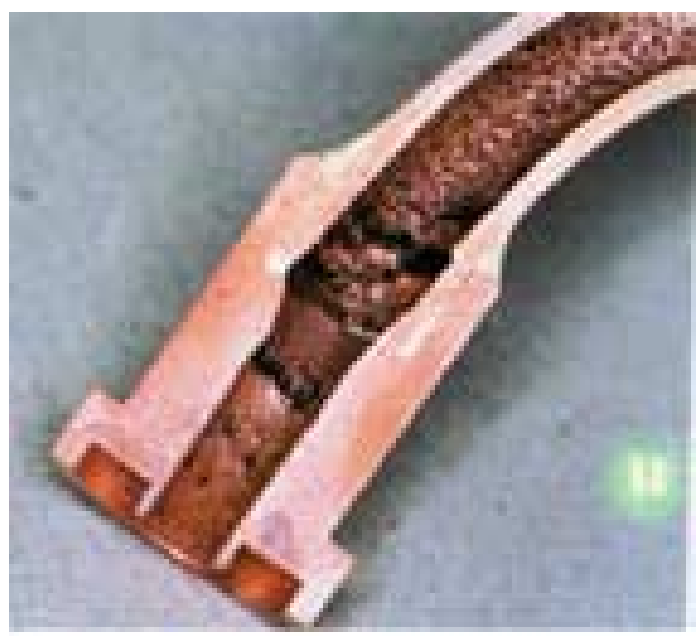

Figure 5: Original step at the welding position becomes too smooth. Water flows from upper to bottom.

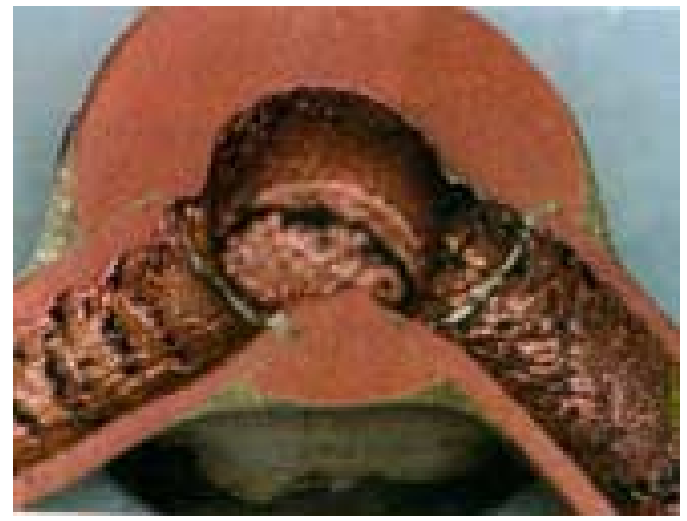

Figure 6: Evidence of the erosion by cavitation. Water flows from right to left.

The water in P11 cooling system was inspected. The density of the tritium was $2.3 \mathrm{~Bq} / \mathrm{cc}$. Negative chlorine ion was $0.06 \mathrm{mg} / \mathrm{l}$ after filtration of $0.45 \mu \mathrm{m}$ filter by ion chromatography. According to the frameless atomic absorption spectrum inspection, amounts of iron, copper and zinc were 4 (22) mg/l, 8 (9) $\mathrm{mg} / \mathrm{l}$ and 14 (24) $\mathrm{mg} / \mathrm{l}$, respectively. The number in brackets is the measured value before filtering. We did not expect that the chlorine ion was remained. It indicates that the chlorine ion is not removed sufficiently by the ion exchange. Chlorine might have caused corrosion.

\section{CONCLUSION}

We have practiced only allopathic at the pinhole leak and/or some other cases of cooling water troubles and the exact reason of the trouble is not yet certain. The cooling system design in the mechanical support building is conforms to basic rules. At the loads, however, the rules are not observed thoroughly and various materials such as brass, copper, iron, stainless steel, plastic and rubber are used. We should use the unified materials as much as possible to mitigate the corrosion. Further, on the assumption that the erosion-corrosion is inevitable in a cooling system, simple and quick washing devices, such as local filtering and flashing, should be equipped at the end of load. In our case of small piping parts for quadrupole magnets and cooling pipe of extraction septa, speed of water flow becomes $6 \mathrm{~m} / \mathrm{s}$ and there are dangerous of erosion such as cavitation, then the flow speed should be reduced as slow as possible in the allowance of the cooling quality. We cannot ignore that the waterweed was found on the inner surface of the P20 cooling system. The visual flow meters using float are equipped. It should be dimly lighted or off the light at unnecessary time. For a future high intensity accelerator, maintenance work should be difficult due to radioactivity and hence careful investigation and selection of the parts for cooling system in the accelerator tunnel are necessary.

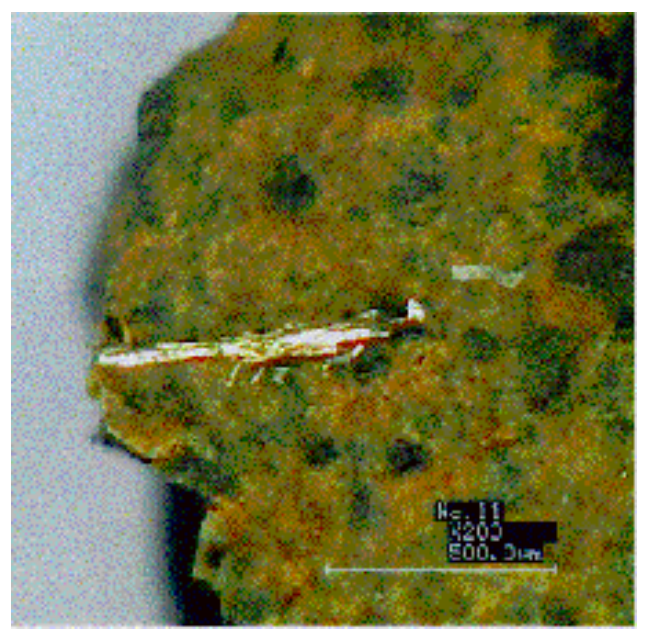

Figure 7: Inner surface of the rubber tube for the field monitor bending magnet.

\section{REFERENCES}

[1] Christine R. Ader et.al., 2000 ASME/JSME Pressure Vessels and Piping Conference, July 23-27, 2000,

Seattle, Washington

[2] P.G.Hurh et.al., PAC99, p.1423

P.G.Hurh , MI-0254, March 4, 1999

[3] M. White et.al., PAC99, p.1414 